\section{Methodenkritische Betrachtung zur Rheobasendiagnostik}

\section{P. Kochenstein}

Fachbereich Psychologie und Pädagogik der Universität Regensburg, Lehrstuhl für Psychologie VI (Direktor: Prof. Dr. H. Lukesch)

Ein neues Verfahren zur Diagnose einer Frühgeburtsgefährdung wurde gefunden und wird propagiert $(12,13)$ : »Es ist daher eine breitere Anwendung der Rheobasenmessung bei allen Frauen in der Frühschwangerschaft als ScreeningMethode zur Früherfassung eines Frühgeburtenrisikos und bei der Behandlung einer drohenden Frühgeburt für die Prognose des weiteren Schwangerschaftsverlaufs sehr zu empfehlen « (12, S. 153).

Rheobase bezeichnet man als diejenige Reizstromstärke, die zur Auslösung einer Minimalzuckung eines quergestreiften Muskels bei einer definierten Reizdauer erforderlich ist.

Folgt man einem Vorschlag von Jung und Mülbert aus dem Jahr 1969, so läßt sich die Rheobasenmessung durch folgende Versuchsanordnung durchführen: Eine $3 \times 3 \mathrm{~cm}$ große differente Elektrode (Kathode) wird am motorischen Punkt des M. tibialis anterior auf gut durchfeuchteter Schwammschicht von der Größe der Silberbleche mit Gummibändern befestigt. Die indifferente $10 \times 15 \mathrm{~cm}$ große Elektrode liegt proximal vom Kniegelenk auf der Vorderseite des Oberschenkels. Die Stromstärke wird so lange gesteigert, bis die Reizschwelle eben überschritten und ein Minimalerfolg der Reizung (Zukkung) feststellbar ist.

Diese Versuchsanordnung wurde im wesentlichen bei einigen späteren Untersuchungen in der gynäkologischen Forschungspraxis beibehalten $(9,17)$ und zum Teil auch modifiziert. Seit 1973 wird zur Objektivierung der Muskelreaktion ein ZweiKanal-Schreiber verwendet (16). Fendel u. Mitarb. schlagen neben der Verwendung eines 1-Kanal-Myographen eine Reizung des M. extensor brevis über den Nervus fibularis vor (7). Feichtinger $\mathrm{u}$. Mitarb. wiederum nehmen den M. tibialis anterior für die Messung, den sie im Gegensatz zu den anderen Untersuchern bipolar mit einer Gabelelektrode (Elektrodengröße $\left.3 \mathrm{~cm}^{2}\right)$ reizen $(3,4,5,6,18)$.

Die einzelnen Untersuchungen sind so nur bedingt miteinander vergleichbar. Ihnen gemein ist jedoch die Aussage, daß Zusammenhänge zwischen neuromuskulärer Erregbarkeit ermittelt durch einen Rheobasenscore - und einem Frühgeburtsrisiko existieren und daß diese Art der Diagnostik zusätzlich Rückschlüsse auf den therapeutischen Verlauf bei der Behandlung von frühgeburtsgefährdeten Schwangeren und für die weitere Prognose zuläßt.

Die für eine Diagnose elementar wichtigen Fragen nach einer Meßreliabilität, Validität und Meßobjektivität werden jedoch

\section{Zusammenfassung}

Die Verwendung eines Rheobasenwertes zur Abschätzung einer Frühgeburtsneigung erscheint uns zur Zeit noch nicht ausreichend gesichert. Die Bestimmung der Rheobase ist in jedem Einzelfall mit so vielen Schwierigkeiten verbunden, die Úbertragbarkeit einer Erregbarkeit von der Skelettmuskulatur auf die glatte Muskulatur der vegetativ innervierten Organe so wenig theoretisch und empirisch bewiesen, daß wir in dieser Technik keinen wissenschaftlichen Fortschritt erblicken können. Diese Behauptungen werden zur Zeit durch weitere Untersuchungen an Schwangeren überprüft.

Die Verwendung eines Rheobasenwertes zur Erkennung einer drohenden Frühgeburt und zur Kontrolle der Effektivität einer tokolytischen Behandlung wird kritisch erörtert. Zahlreiche Erkenntnisse aus elektrodiagnostischen Untersuchungen schränken den Wert dieses Verfahrens in der Geburtshilfe von vornherein ein.

Offenkundige Unzulänglichkeiten bei der RheobasenMessung, insbesondere mangelnde Reliabilität sowie ein bisher nicht kontrollierter möglicher VersuchsleiterEffekt mahnen zu einer vorsichtigen Dateninterpretation. Diese kritische Sichtweise wird mit Daten aus eigenen Experimenten illustriert.

\section{Some Critical Remarks to Rheobase-Diagnosis}

The application of a rheobase-score as a mean to recognize threatening premature labour or to check the effectiveness of a tocolytic treatment is critically discussed. Numerous findings in the course of electrodiagnostical investigations restrict the value of this procedure in obstetrics. Various difficulties with the measurement of rheobase, especially a deficient reliability as well as a hitherto uncontrolled possible experimenter-effect remind of a careful interpretation of data. To illustrate this critical point of view own experiments are reported.

in diesen Untersuchungen nach unserer Ansicht zu wenig behandelt. Unberücksichtigt blieben dabei zudem die Erfahrungen älterer Autoren mit elektrophysiologischen Messungen und die Schwierigkeiten, die sich bei der Gewinnung und Interpretation solcher Daten zwangsläufig ergeben.

Dieses Defizit auszugleichen und gleichzeitig die von Jung (1980) vorgeschlagene Screening-Methode in die klinische Praxis zu implantieren, war somit das erklärte Ziel unserer Untersuchungen ${ }^{1}$ ).

\footnotetext{
$\left.{ }^{1}\right)$ Für die Unterstützung bei diesem Vorhaben danken wir insbesondere Herrn Dr. K. Krumbacher, Chefarzt der Klinik St. Hedwig, Regensburg. Ferner gilt unsere Anerkennung der Firma Siemens, die uns unbürokratisch ein für eine solche Diagnostik brauchbares Gerät (Neuroton 726) zur Verfügung stellte.
} 


\section{Zur Reliabilität der Rheobasendiagnostik}

Bevor ein Meßergebnis sinnvoll interpretiert werden kann, ist es absolut notwendig, den bei jeder Messung unvermeidbaren Fehler zu schätzen und bei der Besprechung der Daten zu berücksichtigen. Der Nachweis einer Ergebniskonstanz unter Beibehaltung der Meßbedingungen wurde in den gynäkologischen Fachveröffentlichungen nicht geführt, d.h. es wurde davon ausgegangen, da $\beta$ irgendwelche Unterschiede in den Rheobasen-Werten bereits auf die Einflüsse wie Medikation oder Zeitpunkt der Schwangerschaft zurückzuführen seien. $\mathrm{Da} B$ diese Meßabweichung oft in der erwarteten Richtung erfolgte, d.h. z. B., daß die Rheobase meist unter PartusistenBehandlung anstieg (wenn auch erst nach Tagen), war für die Untersucher ein weiterer Beleg für die Richtigkeit ihrer Theorie. Zwar wurde stets zusätzlich zum Mittelwert der Messungen ein $\mathrm{Maß}$ für die Streuung der Werte angegeben; die Ursachen für diese Abweichungen wurden jedoch nicht mit einem möglichen Meßfehler in Zusammenhang gebracht, sondern, wenn überhaupt, dann als individuelle Verschiedenheit der Untersuchten in den zur Debatte stehenden Variablen erklärt.

Ein solcher Schluß ist jedoch unzulässig und oftmals Ursache zahlreicher Pseudo-Ergebnisse. Nachfolgend seien einige Arbeiten aufgeführt, die einen solchen Umgang mit den Rheobasenmeßwerten höchst fragwürdig erscheinen lassen. So weisen bereits Neoussikine und Abramowitsch (1939) auf eine Vielzahl von Schwierigkeiten bei der Messung der Rheobase hin: „Um Werte zu bekommen, die eventuell wiedergefunden werden könnten, muß man mit ein und derselben Elektrodengröße arbeiten, die Elektrode auf denselben bestimmten Punkt und mit gleicher Kraft andrücken. Es muß hier gesagt werden, daß eine ganz geringe Verschiebung der Reizelektroden oder kleine Änderung im Anlegen derselben mit verschiedenen Reizschwellenwerten einhergehen können. Es ist hier besonders wichtig, die motorischen Punkte des Muskels und die Nervenpunkte möglichst exakt finden zu können. ...es gehört dazu eine bestimmte Ưbung « (15, S. 74) und weiter: "Die Erfahrung zeigt, daß, wenn man bei der Feststellung der Reizschwelle mehrmals nacheinander reizt, man im Laufe der Untersuchung Schwankungen der Schwellenwerte konstatieren kann « (S. 75).

Drei Dinge, die für die Reliabilität der Messung sicherlich von Bedeutung sind, werden hier angesprochen:

1. Alle technischen Parameter müssen konstant gehalten werden (Elektrodengröße, Druckstärke).

2. Es ist wichtig, einen definierten Punkt (motorischer Punkt) genau zu treffen.

3. Auch dann ergibt ein mehrmaliges Reizen (Messen) unterschiedliche Schwellenwerte.

$\mathrm{Zu}$ diesem letzten Ergebnis kommt auch Kulawik (1965), der die Unzulänglichkeit der Rheobasen-Bestimmung u.a. damit begründet: »...man fand bei wiederholter Reizung unterschiedliche Werte... « $(14$, S. 8$)$ und weiter: »Für praktische Belange ist es wichtig zu wissen, daß bei Rechteckimpulsen zwischen 1000 und $1 \mathrm{msec}$ (mit diesen Impulszeiten erfolgt die Rh.-Diagnostik; Anm. d. Verf.) die Schwellenintensität des normalen Nerv-Muskel-Systems unabhängig vom Niveau der Kurve (gemeint ist die i/t-Kurve bei Normalpersonen, Anm. d. Verf.) Schwankungen bis zu $3 \mathrm{~mA}$ aufweisen kann, wie wir sie bei unveränderten äußeren Bedingungen (serienmäßiger Prüfung) gefunden hatten... Weiterhin ist zu beachten, daß die Schwankungen im Bild der Kurve um so deutlicher in
Erscheinung treten, je niedriger der Rheobasenwert und damit das Niveau der Kurve ist, « »...nur Abweichungen, die deutlich die hier gefundenen Streuwerte überschreiten, können z.B. auf therapeutische Einflüsse bezogen werden. Schwankungen der Schwellenintensität im Bereich zwischen 1000 und $1 \mathrm{msec}$ sind bei Rechteckimpulsen nur dann diagnostisch verwertbar, wenn sie deutlich größer (Hervorh. d. d. Verf.) als $3 \mathrm{~mA}$ sind « $(14$, S. 43 f., S. 47).

Auch Edel weist in diesem Zusammenhang auf die Streubreite der Schwellenwerte hin:

»Bei der Beurteilung der Normalwerte der einzelnen Muskeln und Nerven sollte man die relativ große biologische Streubreite von einer zur anderen Untersuchungsperson nicht vergessen... wir sind aber der Meinung, da $\beta$ auch bei der gleichen Person bei der RIC Schwankungen der Schwellenintensität im Bereich zwischen 1000 und $1 \mathrm{~ms}$ nur dann diagnostisch verwertbar sind, wenn sie mindestens über $3 \mathrm{~mA}$ liegen $\approx$ (2, S. 855). Die alleinige Bestimmung der Rheobase wird hier im Vergleich zur Ermittlung anderer elektrophysiologischer Werte (Chronaxie, Akkommodabilität) als die am wwenigsten aussagekräftige « herausgestellt. Zusammenfassend schreibt er in seiner »Fibel der Elektrodiagnostik und Elektrotherapie « (1973): „Die Streubreite der Rheobasenwerte um den Mittelwert ist aber bei verschiedenen Personen nicht unerheblich, wie wir uns in einigen Untersuchungen unter standardisierten Bedingungen überzeugen konnten. So betrug der getestete Mittelwert der Rheobase für den M. ext. dig. c.: $\bar{X}=3,2 \mathrm{~mA}, \mathrm{~S}= \pm 1,2 \mathrm{~mA}$, der 1-s-Streubereich reicht aber von 2 bis $4,4 \mathrm{~mA}$, der in der Biologie meist benutzte 2 -s-Streubereich von 5,6 bis $0,8 \mathrm{~mA}$. Es können also nur erhebliche Abweichungen etwas aussagen... « (1, S. 43). Zum selben Ergebnis kommen Jantsch und Schuhfried (1974): »Eine Angabe der Höhe der Rheobase in $\mathrm{m} \mathrm{A}$ allein ist nur von geringer Aussagekraft... Die eigentliche Bedeutung der Rheobase liegt in der Festlegung eines Grundwertes, auf dem sich die Bestimmung der Akkommodationsfähigkeit und der Chronaxie aufbauen « $(8, \mathrm{~S} .81)$.

Desgleichen auch Harris (Ưbers. d. d. Verf.): „Die Rheobasen-Werte streuen weit in den verschiedenen Muskeln und besitzen eine hohe individuelle Variabilität« (3, S.231). Extremwerte sollten nicht interpretiert werden, bevor die verwendete Meßtechnik perfekt sei, und selbst dann sei die isolierte Angabe der Rheobase für eine Diagnose und Prognose nutzlos.

\section{Mögliche Einflußfaktoren auf die Rheobasenbestimmung}

Wie kommt es aber zu solchen oft extremen Schwankungen bei dieser Diagnostik? Welche Faktoren sind für diese Streubreite verantwortlich zu machen, bzw. gibt es überhaupt einen Schwellenstromwert, den man sinnvollerweise als Rheobasenwert bezeichnen kann?

Alle genannten Autoren betonen stets, wie wichtig es für die Ermittlung des Rheobasenwertes sei, daß die Reizelektrode (Kathode) genauestens am sog. motorischen Punkt aufliegt. Das bedeutet, daß es unumgänglich ist, diesen Muskelreizpunkt, der bei einem normal innervierten Muskel der Eintrittsstelle des motorischen Nervs in den Muskel entspricht und somit anatomisch festgelegt ist, vor jeder Messung zunächst einmal auszumachen. Das kann jedoch im Einzelfall einen großen Aufwand an Zeit und Mühe kosten (bis zu $30 \mathrm{~min}$ ), wie sich der Verfasser selbst überzeugen konnte. Die für diese Arbeit zur Verfügung stehende Zeit steht jedoch in 
direktem Zusammenhang mit der Höhe des ermittelten Rh.Wertes nach folgender einfacher Überlegung: Je größer der aufgebrachte Zeitaufwand ist, um so größer ist die Wahrscheinlichkeit, daß man auch wirklich diesen motorischen Punkt erfaßt hat. Je näher man diesen Punkt mit der Reizelektrode trifft, um so niedrigerer Stromstärken bedarf es, um eine Muskelzuckung sichtbar zu machen $-\mathrm{d}$. h. um so geringer ist der gemessene Rh.-Wert.

Die Schwankungen sind also möglicherweise hauptsächlich auf die unterschiedliche Sorgfalt bei der Messung durch die Experimentatoren zurückzuführen bzw. dadurch entstanden, daß man bei jeder Messung diesem motorischen Punkt verschieden nah gekommen ist.

Ein weiterer Sachverhalt kompliziert die Diagnose eines Rheobasenwertes:

Zumindest in den älteren Schriften zur Elektrodiagnostik finden sich Tabellen und Skizzen, die für verschiedene Muskeln - so auch für den oft untersuchten M. tibialis anterior mehr als nur einen Muskelreizpunkt (meist 2-3) mit jeweils verschiedenen Schwellenwerten anführen. Die Konsequenz für eine exakte Rheobasendiagnostik ohne genaueste Angabe der Vorgehensweise ist offensichtlich. Die millimetergenaue Plazierung der Reizelektrode ist also für die Meßtechnik von wichtigster Bedeutung. Daneben werden jedoch verschiedene andere Variablen genannt, die für die Diagnose der Schwellenwerte Rheobase und der daraus abgeleiteten Messung der Chronaxie relevant sind.

Unter Chronaxie versteht man die Impulsdauer (in msec), die für eine eben sichtbare Muskelzuckung erforderlich ist, wenn man mit einem Strom von doppelter Rheobasenstärke reizt. Ihre Bestimmung setzt also zunächst die Ermittlung der Rheobase voraus. Allgemein gilt die Chronaxie im Vergleich zur Rheobase als die weniger störbare Meßgröße $(4,8)$, d. h. wenn Faktoren auf die Chronaxie-Messung Einfluß nehmen, kann dies erst recht für die Rheobase-Diagnose angenommen werden. Die folgenden genannten Einflußfaktoren wurden teils für die Rheobase, teils für die Chronaxiemessung postuliert, wobei die Vielzahl der in der Literatur für die Schwellenwertbestimmung benannten Wirkgrößen eine große Meßvarianz wahrscheinlich macht.

Als technische Parameter wurden bereits die Elektrodengröße und die Aufdruckstärke der Reizelektrode erwähnt. Von den eher biologischen Variablen werden u. a. folgende genannt:

1. Zustand der Haut am untersuchten Muskel: Entwicklung der Epidermis, subkutanes Zell- und Fettgewebe, Grad der Feuchtigkeit, Anwesenheit einer größeren Zahl von Schweißund Fettdrüsen, Hautdurchblutung, Hauttemperatur.

2. Zustand des untersuchten Muskels: Dehnung, Ermüdung, allg. Körperhaltung (Unterschied bspw. ob Bauch- oder Rückenlage), Durchblutung.

3. Blut: $\mathrm{pH}-$ Wert, u. a. Adrenalin-, Insulin-, $\mathrm{CaCl}_{2}-$ Spiegel, Vitamin A-, Alkohol-Gehalt im Blut.

4. Psychische Faktoren: emotive Einflüsse (Schreck), intellektuelle Arbeit, Anstrengung, Aufmerksamkeit, Suggestion.

All diese Größen, hier nur auszugsweise wiedergegeben, wurden in Zusammenhang mit den physiologischen Schwellenwerten Rheobase und Chronaxie gebracht. Dies könnte zum Nachdenken anregen - auch dann, wenn diese »nachgewiesenen « Einflußfaktoren nicht in jeder Untersuchung bestätigt werden konnten. Eine Konstanthaltung all dieser Bedingungen erscheint auf jeden Fall ein Ding der Unmöglichkeit.
Doch zurück zur Meßobjektivität: Rheobase als die Schwellenstromstärke für eine eben sichtbare Muskelzuckung mag wohl zusätzlich von den Beobachtungskriterien bei der Messung abhängen. »Als Kriterium einer Minimalzuckung wird die eben sichtbare, seltener die eben tastbare (Anspannung einer Sehne) Muskelzuckung bei Erreichen der Schwellenstromstärke bezeichnet (Subjektives Moment!)« (2, S. 853). Was nun? Wie genau ist »eben sichtbar, eben tastbar «? Ganz offensichtlich können hier Meßunterschiede, hervorgerufen evtl. durch persönliche Variable des Untersuchers (z. B. seine Motivation, Erwartungshaltung), auftreten und die Interpretation der Ergebnisse erschweren.

Die Objektivierung der Ablesung der Muskelzuckung durch Verwendung eines Myographen erscheint dabei als PseudoFortschritt, da erstens auch hier die Interpretation der Reizantwort (Zuckung) normiert werden muß und von subjektiven Faktoren (Ablesungsgenauigkeit) abhängig ist und zweitens man ja in diesem Fall gar nicht mehr von Rheobase im eigentlichen Sinn (vgl. Def.) sprechen kann.

Durch Verwendung einer Nadelelektrode mit Oszillograph erhielten wir für die Rheobase einen Wert von kleiner als $1 \mathrm{~mA}$ - einen Wert also, der jenseits aller Vergleichbarkeit mit den sonst zitierten Stromstärken steht. Ein größerer technischer Aufwand ist in diesem Fall nicht mit einer Verbesserung der diagnostischen Objektivität verbunden. Je genauer ich die Messung vornehme (Nadelelektrode, Myogramm), um so eher erhalte ich bereits bei minimalsten Stromstärken elektrophysiologische Reaktionen - mit Rheobasenmessung hat das dann jedoch immer weniger zu tun. Der ermittelte Schwellenwert der Stromstärke hängt auf jeden Fall aufs engste mit der gerade verwendeten Meßmethode, mit den zur Verfügung stehenden Meßgeräten und mit großer Wahrscheinlichkeit auch mit den Persönlichkeitsvariablen des Untersuchers zusammen.

All diese Schwierigkeiten lassen eine Verwendung der Rheobasenmessung zur Diagnose einer Frühgeburtsgefährdung höchst fraglich erscheinen. Der theoretische Hintergrund für diese Diagnostik sei im Zusammenhang mit der Validitätsfrage kurz dargestellt: Es wird angenommen, daß irgendwelche Verbindungen zwischen neuromuskulärer Erregbarkeit festgestellt durch die Höhe des gemessenen Rheobasewertes und Geburtsbereitschaft des Uterus existieren. »Wir hatten uns damals der medizinischen Grundlagenkenntnisse erinnert, $\mathrm{da} ß$ vegetativ innervierte Organe wie der Darm und der Uterus über zentrale Schaltneurone des Rückenmarks eine Beziehung zu motorischen Neuronen der quergestreiften Muskulatur und zur Haut besitzen«, schreibt Jung (1981).

Angeregt zur Annahme einer solchen Beziehung wurden Jung und Mülbert (1969) durch folgende Beobachtung: »Für eine Stichprobenuntersuchung des mittleren Rheobasenabfalls bei $400 \mathrm{msec}$ zwischen schwangeren und nichtschwangeren Frauen gegenüber den Werten unter der Geburt ist die Differenz nach dem Student-Test bei einem p $<0,001$ statistisch signifikant « (11, S. 233f.). Diese Feststellung wurde an einer Stichprobe von 30 schwangeren Frauen während der Eröffnungsperiode, verglichen mit 30 Schwangeren, die während der 33. und 37. Schwangerschaftswoche gemessen wurden, und 30 nicht schwangeren Frauen getroffen.

Der enge Zusammenhang zwischen Rheobase und Wehentätigkeit wird weiter aufgrund einer ca. 20tägigen Verlaufsbeobachtung an einer 31jährigen Schwangeren dokumentiert, bei der immer wieder das spontane Auftreten von leichten Wehen von einem Abfall der Rheobase begleitet war - auch eine 
Amnioskopie hatte einen prompten Rheobasenabfall zur Folge.

Deutlich relativiert wird diese Beziehung jedoch in einer Arbeit von Tietze (17, S. 35): „Die Kombination von Wehenregistrierungen im Kardiotokogramm und pathologischen Rheobasenwerten, d.h. der Wert liegt unter $4 \mathrm{~mA}$, konnte in 99 Bestimmungen nachgewiesen werden. Dies entspricht $45 \%$ der Messungen. Bei 120 Bestimmungen der Rheobase wurde ein pathologischer Wert ermittelt, ohne daß es gelang, gleichzeitig eine Wehentätigkeit aufzuzeichnen. Eine Beziehung zwischen Wehentätigkeit und erniedrigter Rheobase konnte also in 55\% der Messungen nicht hergestellt werden. «

Die Rheobasendiagnostik ersetzt somit keine kardiotokographische Untersuchung. Die Jungsche Beobachtung könnte also entweder ein zufälliges Ergebnis sein oder darauf beruhen, daß in den Fällen, in denen eine Wehentätigkeit festgestellt bzw. eine Amnioskopie vorgenommen wurde, auch mit größerer Sorgfalt der Rheobasenwert gemessen wurde.

Was hier im allgemeinen zur Rheobasendiagnostik ausgeführt ist, gilt möglicherweise ganz speziell immer dann, wenn mit ihrer Bestimmung ein Frühgeburtsrisiko abgeschätzt werden soll: Je pathologischer der Befund (Anamnese, Zervixbefund, Wehentätigkeit) in Richtung Frühgeburtlichkeit weist, um so größer mag die bei der Messung waltende Genauigkeit und Sorgfalt der Untersucher anzusetzen sein. Es ist anzunehmen, $\mathrm{da} B$ zumindest nicht in jedem Fall der Rheobasendiagnostiker von den übrigen medizinischen Daten der untersuchten Schwangeren nichts wußte. Dies ist schon deshalb kaum möglich, da viele der diesbezüglichen Messungen stationär durchgeführt wurden. Eine gewisse Erwartungshaltung des Untersuchers in bezug auf das Meßergebnis ist somit stets als Fehlerquelle mit zu berücksichtigen.

$\mathrm{Zu}$ der vermuteten Beziehung zwischen neuromuskulärer Erregbarkeit und Uterusmotilität herrscht auch in theoretischer Betrachtung keine klare Sicht. Jung (11) beruft sich auf einen bekannten »Reflexweg « zwischen vegetativen Organen, der Haut und dem Skelettsystem, weist aber gleichzeitig darauf hin, $\mathrm{da} B$ über die Rolle des vegetativen und zentralen Nervensystems für die Uterusmotilität und Geburtsauslösung wenig bekannt sei.

Nach unserer Auffassung ist ein Zusammenhang zwischen Frühgeburtsbestrebungen und Rheobasenwert noch nicht hinreichend gesichert, um aus der Bestimmung des letzteren eine Aussage über ein Frühgeburtsrisiko treffen zu können.

Die mit dieser Fragestellung befaßten Arbeiten stehen vor einer zusätzlichen fast unlösbaren Schwierigkeit.

Drei Gruppen von untersuchten Schwangeren werden meist gegenübergestellt und hinsichtlich ihrer Daten miteinander verglichen: »Normal«-Schwangere, Risiko-Schwangere mit vermiedener Frühgeburt und Schwangere, bei denen es zu einer Frühgeburt kam.

Unklar bleibt meist, nach welchen Kriterien es zu der Einschätzung »Risiko-Schwangere « kam, ob dabei der ermittelte Rheobasenwert eine zuweisende Rolle gespielt hat und welche $\mathrm{Maßnahmen} \mathrm{im} \mathrm{Einzelfall} \mathrm{gegen} \mathrm{das} \mathrm{Risiko} \mathrm{getroffen} \mathrm{wurden.}$ Welche von der Rheobasendiagnostik unabhängigen Kriterien einer drohenden Frühgeburt wurden mit welcher Gewichtigkeit für die Untersuchungen herangezogen?

Eine der möglichen $\mathrm{Maßnahmen,} \mathrm{die} \mathrm{mit} \mathrm{gutem} \mathrm{Grund} \mathrm{gegen}$ drohende Frühgeburtsbestrebungen durchgeführt werden könnte, ist die Anwendung eines Entspannungstrainings zur allgemeinen Ruhigstellung des Organismus.
Herms (9) überprüfte bei 89 Schwangeren die Wirkung eines solchen Trainings anhand des Respiratorischen Biofeedback einer Methode, bei der die Versuchsperson lernt, über die Rückmeldung der Atemexkursionen zahlreiche vegetative Funktionen zur Relaxation anzuregen.

Neben einigen beeindruckenden Ergebnissen - signifikante Abnahme der Wehentätigkeit und des Blutdrucks (bei Frauen mit EPH-Gestose) im prä/post-Vergleich - wird auch eine Wirkung des Trainings auf die Rheobase berichtet: „Bei den 28 Patientinnen der Studie 4.4.2 wurden jeweils 3 Messungen vor und nach der Behandlung mit dem RFB durchgeführt.

vorher

$\mathrm{N}=84$ Messungen

$\overline{\mathrm{X}}=4,2$

nachher

$\mathrm{S}^{2}=1,96$

$\overline{\mathrm{X}}=5,0$

$\mathrm{S}=1,4$

$\mathrm{S}^{2}=2,56$

$\mathrm{S}=1,6(\mathrm{p}=0,05)$

Durch die Behandlung mit dem RFB kommt es zu einem signifikanten Anstieg der Rheobase. Möglicherweise ist dieser Anstieg durch eine Veränderung des galvanischen Hautwiderstandes mitbeeinflußt « $(9$, S. 96)

Bemerkenswert erscheint hierbei, daß man Ergebnisse in der erwarteten Richtung (Abnahme neuromuskulärer Erregbarkeit) erzielte und gleichzeitig die Möglichkeit einer weiteren Wirkgröße in Betracht zieht. Ohne auf die Problematik dieser Interpretation näher einzugehen, sei an dieser Stelle ein Experiment berichtet, das wir zur Uberprüfung dieser Frage (Zusammenhang Rheobase/Entspannung - Erwartungshaltung) durchführten: Die Untersucher (Studenten der Anfangssemester) wurden angewiesen, die Wirkung eines Entspannungstrainings (progressive Muskelrelaxation nach Jacobson) auf die Rheobase festzustellen. Vor den Experimenten wurde dabei folgende Suggestion gegeben: ein entspannter Organismus reagiert sensibler auf Außenreize. Diese gesteigerte Sensibilität geht konform mit einer höheren Leistungsbereitschaft des gesamten motorischen und kognitiven Systems. Die Werte der Rheobase werden voraussichtlich nach der Entspannung erniedrigt sein.

Gemessen wurde eine Gruppe von Studenten; 5 männliche, 6 weibliche, Alter zwischen 20 und 32 Jahren; die Untersucher wurden mit der Meßtechnik im Eigenversuch vertraut gemacht.

Ergebnis:

vor der Entspannung nachher

$\overline{\mathrm{X}}=8,91 \quad \overline{\mathrm{X}}=7,18$

$\mathrm{S}=2,95 \quad \mathrm{~S}=1,78$

Test für abhängige Messungen $=3,68^{++}$

krit. $1 \%(\mathrm{df}=10)=2,76$.

Die Ergebnisse zeigen also auch hier auf hohem Signifikanzniveau in die erwartete (suggerierte) Richtung und stehen zu der Untersuchung von Herms im Widerspruch.

Uns ging es bei diesem Experiment in erster Linie um den Effekt einer Erwartungshaltung auf die Ergebnisse - keinesfalls sind wir geneigt, aufgrund dieser Untersuchung eine tatsächliche Steigerung der neuromuskulären Erregbarkeit nach einem Entspannungstraining anzunehmen. Hierfür müßten eine Vielzahl weiterer kontrollierter Studien durchgeführt und deren Ergebnisse herangezogen werden.

Eine weitere Einzelbeobachtung sei hier erwähnt: Im Verlauf unserer Experimente untersuchten wir eine Kollegin und stellten nach sorgfältiger Messung einen Rheobasenwert von 3,3 mA fest. Eine von einer Ärztin im Rahmen einer Schwangerschaftsvorsorgeuntersuchung an derselben Probandin 
wenige Wochen später durchgeführte Messung ergab den Rheobasenwert $10 \mathrm{~mA}$.

Verständlicherweise sind solche Meßwertdifferenzen für uns ein Anlaß, die Rheobasendiagnostik mit größerer Skepsis zu betrachten.

Abschließend sei im Hinblick auf die von Jung (12) postulierte pathologische Grenze von $4 \mathrm{~mA}$ ein Zwischenergebnis unserer Untersuchungen an 20 nicht schwangeren Frauen referiert, die mit dem Anspruch durchgeführt wurden, alle möglichen Fehlerquellen bei der Messung so gering wie möglich zu halten.

\section{Literatur}

(1) Edel, H., Fibel der Elektrodiagnostik und Elektrotherapie. 2. Aufl. Verl. Theodor Steinkopff, Dresden 1973

(2) Edel, H., Klinische Elektrodiagnostik des peripheren Nervensystems unter besonderer Berücksichtigung der Reizstromdiagnostik. Zschr. ärztl. Fortbild. 61.Jg. 17 (1967) 852-858

(3) Feichtinger, W., H. Fröhlich, Elektrodiagnostische Ermittlung von Reizzeit-Intensitätskurven während der Schwangerschaft, unter der Geburt und im Wochenbett sowie deren Beurteilung. Z. Geburtsh. u. Perinat. 183 (1979) 359-363

(4) Feichtinger, W., H. Fröhlich, Elektrodiagnostische Untersuchungen während der Schwangerschaft, unter der Geburt, im Wochenbett und bei drohender Frühgeburt. Z. Geburtsh. u. Perinat. 183 (1979) 364-368

(5) Feichtinger, W., G. Altmann, H. Fröhlich, Neuromuskuläre Erregbarkeit und Geburtsdauer. Rheobase vor und nach Amniotomie zur Geburts- einleitung. Z. Geburtsh. u. Perinat. 184 (1980) 205-208

(6) Feichtinger, W., G. Altmann, Rheobase, Pelvic Score und Oxytocinempfindlichkeitstest vor spontanem Geburtsbeginn. Z. Geburtsh. u. Perinat. 185 (1981) 141-143

(7) Fendel, H., H. Jung, A. Renoldi, Erfahrungen mit einer neuen Methode zur Rheobase-Messung unter der Tokolyse-Therapie mit Fenoterol. In: Jung, H. u. Friedrich, E. (Hrsg.): Fenoterol bei der Behandlung in der Geburtshilfe und Perinatologie.

2. Symposion über Partusisten. Wiesbaden, Oktober 1977. Thieme, Stuttgart 1978

(8) Harris, R., Chronaxy. In: Licht, S. (Hrsg.): Electrodiagnosis and Electromyography. New Haven, 1962, 218-240

(9) Herms, V., Psychosomatische Aspekte vorzeitiger Wehen. Habilitationsschrift, Univ. Heidelberg, Oktober 1980

(10) Jantsch, H., F. Schuhfried, Niederfrequente Ströme zur Diagnostik und Therapie. Verlag Wilhelm Maudrich, Wien - München - Bern 1974

Wir erhielten einen mittleren Rh.-Wert von $\overline{\mathrm{X}}=3,16 \mathrm{mit}$ einer Standardabweichung von $\mathrm{S}=1,12$.

19 der untersuchten Frauen waren danach der Gruppe "Frühgeburtsrisiko zuzuordnen. Nur eine der Frauen erhielt den Wert $7 \mathrm{~mA}$, wobei wir nicht sicher waren, ob wir in diesem Fall genau den motorischen Punkt bei der Messung aufspüren konnten. Die durchschnittliche Zeit für eine Messung lag bei etwa 15 Min.

Auf jeden Fall übten wir bei der Interpretation der Meßergebnisse im Hinblick auf die Frühgeburtsgefährdung unserer Probandinnen größte Zurückhaltung.

(11) Jung, H., S. Mülbert, Neuromuskuläre Erregbarkeit, Wehentätigkeit und Geburtsbeginn. Geburtsh. u. Frauenheilk. 29 (1969) 231-238 (12) Jung, H., Aussagekraft der Rheobasen-Messung für die Routinediagnostik der drohenden Frühgeburt. Deutscher Kongreß für Perinatale Medizin, Berlin 11.-14. Juni 1979. Kongreßbericht Bd. VIII, Thieme, Stuttgart 1981, 151-153

(13) Jung, H., Ursache und Diagnostik der Frühgeburt. Kongreßbeitrag zum Amberger Symposion 9.-11. Oktober 1981. Frühgeburt. Im Druck (14) Kulawik, H., Das Verhalten der i/t-Kurven von Normalpersonen bei wiederholter serienmäßiger Reizung des Nerv-Muskel-Systems. Inauguraldissertation. Med. Akademie »Carl Gustav Carus «, Dresden 1965 (15) Neoussikine, B., D. Abramowitsch, Elektrodiagnostik. Hans Huber, Bern 1939

Dipl.-Psych. P. Kochenstein Psychologische Praxis Lindwurmstraße 135, 8000 München 2
(16) Rüttgers, H., D. Schenk, F. Kubli, Rheobase-Verlaufskontrollen bei Frühgeburtsbestrebungen. In: Jung, H. u. Friedrich, E. (Hrsg.): Fenoterol bei der Behandlung in der Geburtshilfe und Perinatologie. 2. Symposion über Partusisten, Wiesbaden, Oktober 1977. Thieme, Stuttgart 1978

(17) Tietze, R., Die Wertigkeit der geburtshilflichen Anamnese, des Pelvic Score, der Tokographie und der Rheobase-Bestimmung in der Schwangerschaft zur Erkennung der Frühgeburtsbestrebung. InauguralDissertation. Univ. Heidelberg, 1976

(18) Trnavsky, G., W. Feichtinger, H Fröhlich, Grundlagen zur Standardisierung der bipolaren Reizstromdiagnostik in der Geburtshilfe. Z. Geburtsh. u. Perinat. 184 (1980) 41-46 
da der Ausgang der Schwangerschaft zum Zeitpunkt der Rheobasenmessung ja in aller Regel nicht bekannt sein konnte. Allerdings ist richtig und gilt ganz allgemein, daß der Nachweis statistisch signifikanter Unterschiede für einen Meßparameter nicht unbedingt gleichzusetzen ist mit dessen diagnostischer Brauchbarkeit in der Praxis, da für das letztere eine ungleich höhere Trennschärfe notwendig ist als für das erstere. Es gibt zahlreiche epidemiologische, anamnestische und befundmäßige Größen, die mit der Frühgeburt korreliert sind, doch verfügt keine über ausreichende Trennschärfe, um für sich allein praktisch diagnostisch relevant zu werden. Solches war auch für die Rheobase als Ausdruck neuromuskulärer Erregbarkeit weder zu erwarten, noch ist es je behauptet worden. Die Frühgeburt ist ein multifaktorielles Geschehen; die qualitative und quantitative Einschätzung des Frühgeburtenrisikos zu einem Zeitpunkt, zu dem noch mit guten Erfolgsaussichten therapiert werden kann, beruht immer auf einem bewußt oder unbewußt angewandten Risikoscore. Die Rheobasenmessung kann nur Teil davon sein. Es ist aber zuzugeben, daß der Stellenwert der Rheobasenmessung innerhalb des diagnostischen Systems heute noch nicht eindeutig festgelegt ist und daß vor einer breiteren Anwendung in der Praxis wohl vorteilhafterweise noch weitere kontrollierte prospektive Studien stehen sollten.

Prof. Dr. med. F. Kubli

Univ. Frauenklinik

Voßstraße 9, 6900 Heidelberg 